\title{
Metformin inhibition of neuroblastoma cell proliferation is differently modulated by cell differentiation induced by retinoic acid or overexpression of NDM29 non-coding RNA
}

\author{
Delfina Costa ${ }^{1,3}$, Arianna Gigoni ${ }^{1,3}$, Roberto Würth², Ranieri Cancedda ${ }^{1,3}$, Tullio Florio ${ }^{2,4}$ and Aldo Pagano ${ }^{1,3^{*}}$
}

\begin{abstract}
Background: Metformin is a widely used oral hypoglycemizing agent recently proposed as potential anti-cancer drug. In this study we report the antiproliferative effect of metformin treatment in a high risk neuroblastoma cell model, focusing on possible effects associated to different levels of differentiation and/or tumor initiating potential.

Methods: Antiproliferative and cytotoxic effects of metformin were tested in human SKNBE2 and SH-SY5Y neuroblastoma cell lines and in SKNBE2 cells in which differentiation is induced by retinoic acid treatment or stable overexpression of NDM29 non-coding RNA, both conditions characterized by a neuron-like differentiated phenotype.

Results: We found that metformin significantly inhibits the proliferation of NB cells, an effect that correlates with the inhibition of Akt, while AMPK activity resulted unchanged. Notably, metformin effects were modulated in a different ways by differentiating stimuli, being abolished after retinoic acid treatment but potentiated by overexpression of NDM29.
\end{abstract}

Conclusion: These data suggest the efficacy of metformin as neuroblastoma anticancer agent, and support the requirement of further studies on the possible role of the differentiation status on the antiproliferative effects of this drug.

Keywords: Metformin, Neuroblastoma, NDM29 ncRNA, Differentiation, Anti-cancer therapy

\section{Introduction}

Metformin (N,N-dimethylbiguanide) is an oral biguanide in clinical use since the 1950s for its hypoglycemic activity. Currently, it is the most widely used anti-type 2 diabetes drug, with nearly 120 million prescriptions worldwide filled every year [1]. Metformin decreases hyperglycemia primarily by activating 5'-adenosine monophosphate-activated protein kinase (AMPK) to suppress glucose production in the liver, increase glucose utilization, and reduce hyperinsulinemia [2].

Recent population studies suggested that metformin decreases the incidence of several cancers and cancermortality in diabetic patients [3-6], and improves the response to chemotherapy in diabetic breast cancer

\footnotetext{
* Correspondence: aldo.pagano@unige.it

'Department of Experimental Medicine (DIMES), University of Genova, Genova, Italy

${ }^{3}$ IRCCS-AOU San Martino-IST, Genova, Italy

Full list of author information is available at the end of the article
}

patients [7]. Additionally, this therapeutic potential has been confirmed in vitro since metformin inhibits in breast, colon, lung, prostate, and pancreas cancer cell proliferation [8-11]. These studies highlight a direct antitumoral activity of metformin, besides the possible indirect effects mediated by the improvement of the metabolic parameters and, in particular, of the hyperinsulinemia. More recently, prospective studies also demonstrated that preoperative metformin treatment of non-diabetic patients with breast (two weeks) or colorectal aberrant cryptic foci (one month) provided a reduction of the number of proliferative cells $[12,13]$.

Interestingly, it was shown that the antitumor effect exerted by metformin in breast cancer, glioblastoma, and hepatocellular carcinoma cells is mainly mediated by a directed and selective antiproliferative activity against the cancer stem/tumor initiating cell (TIC) fraction [14-17]. According to the cancer stem cell theory this cell subpopulation represents the main pharmacological 
target to obtain efficacious therapeutic responses in tumors [18-20].

In this work we address, for the first time, the possible anticancer effect of metformin in a high risk neuroblastoma (NB) cell model, including cancer cell lines displaying different levels of differentiation and stemness/tumor initiating potential.

In particular, we document a significant inhibition of NB cells proliferation and viability exerted by metformin. Interestingly, overexpression of NDM29, a NB differentiating non-coding (nc)-RNA, transcribed by RNA polymerase III, and able to reduce cell tumorigenicity [21-23], leads to an increased cell sensitivity towards metformin, while all trans-retinoic acid (ATRA)-induced differentiation reduced metformin NB cell susceptibility.

These findings provide the basis for further, deeper investigations on the possible usefulness of metformin as adjuvant/neo-adjuvant treatment for $\mathrm{NB}$, and its specific role in the stemness/differentiation balance of tumor cells.

\section{Materials and methods}

\section{Cell Cultures and metformin treatment}

Cell lines: SH-SY5Y, grown in DMEM (Sigma-Aldrich), supplemented with 10\% FBS (GIBCO), L-glutamine (2 mM; EuroClone), and penicillin-streptomycin $(100 \mathrm{U} / \mathrm{ml} /$ $100 \mu \mathrm{g} / \mathrm{ml}$; EuroClone); SKNBE2, grown in RPMI (SigmaAldrich), supplemented with 10\% FBS (GIBCO), L-gluta mine (2 mM; EuroClone), and penicillin-streptomycin (100 $\mathrm{U} / \mathrm{ml} / 100 \mu \mathrm{g} / \mathrm{ml}$; Euro Clone).

SKNBE2 cells were transfected using polyethylenimine (PEI; Sigma P3143) with pEGFP-N1 as control (hereafter referred to as pMock) or pEGFP-N1-NDM29 (hereafter referred to as NDM29). G418 (geneticin; Invitrogen) was used in culture medium as mean of selection up to $1000 \mu \mathrm{g} / \mathrm{ml}$, until resistant clones were identified. After selection, the clones were preserved in $200 \mu \mathrm{g} / \mathrm{ml} \mathrm{G418} \mathrm{in}$ standard culture conditions. Treatment with metformin (20 mM) was performed when cell culture reached $80 \%$ of confluence. ATRA treatment was performed in SKNBE2 and SHSY5Y neuroblastoma cells grown in RPMI or DMEM medium with $10 \%$ FBS. Cells were grown for 2 days to reach the log phase of growth. When cell cultures reached $80 \%$ of confluence the medium was replaced with RPMI or DMEM medium containing 10\% FBS and ATRA $(1$ or $10 \mu \mathrm{M})$ or DMSO $(0.01 \%$ or $0.1 \%)$ in control cultures. Cells were then grown for 10 days before the experiments were performed.

\section{Cell proliferation and cytotoxicity assays}

A) Real time cell proliferation and cytotoxicity was assessed by xCELLigence RTCA DP System (Roche, Germany), as reported [24]. This system monitors cellular events in real time by measuring electrical impedance across interdigitated gold micro-electrodes integrated on the bottom of tissue culture plates. The impedance measurement provides quantitative information about the biological status of the cells, including cell number, viability, and morphology. Cell-sensor impedance is expressed as an arbitrary unit called Cell Index [25]. In order to calculate $\mathrm{CI}$, cells were seeded into $100 \mu \mathrm{L}$ of standard medium in 96X microtiter plates (E-Plate-Roche, Germany). Background impedance was determined using $50 \mu \mathrm{l}$ of standard medium. After 24 hrs, $20 \mathrm{mM}$ metformin was added to the wells and cell proliferation was monitored for 72 hrs or more. Cell adhesion, spreading and proliferation were monitored every $30 \mathrm{~min}$ using the xCELLigence system to produce time-dependent cell response dynamic curves. All experimental results were obtained using RTCA Software 1.2 of the xCELLigence system.

B) Cell counting studies: cell from the different lines were plated in 6-well plates, incubated in standard medium for approximately $24 \mathrm{hrs}$ before being treated with $20 \mathrm{mM}$ metformin. Cells were counted with a hemocytometer after 24 and 48 hrs of metformin treatment and evaluated by Trypan blue exclusion test in which live cells are able to exclude the dye from the cytosol.

C) $\left[{ }^{3} \mathrm{H}\right]$-thymidine incorporation assay: different cell lines were plated in 24-well plates, incubated in standard medium for $24 \mathrm{hrs}$, then treated with $20 \mathrm{mM}$ metformin. After 24 and $48 \mathrm{hrs}$ of metformin treatment, cells were pulsed with $\left[{ }^{3} \mathrm{H}\right]$-thymidine $(0.3 \mu \mathrm{Ci} / 500 \mu \mathrm{l} /$ well $)$ (GE Healthcare, New York, NY) for the last 14 hrs. Averaged proliferation rate was then calculated by the thymidine uptake assay.

D) ATPlite 1step Luminescence assay: this system measures cellular ATP levels, as a marker of cell viability (Perkin Elmer, Monza, Italy). ATPlite 1step assay system is based on the production of light caused by the reaction of ATP with added luciferase and D-luciferin. The emitted light is proportional to ATP concentration. Cell suspension $(100 \mu \mathrm{l})$ was seeded in 96 -well culture plate white (Thermo Scientific). After $24 \mathrm{hrs}, 100 \mu \mathrm{l}$ culture medium containing metformin $(20 \mathrm{mM})$ was added to the cells. Each group had 5 repeats. The plates for the ATPLite assay were incubated for 24 or $48 \mathrm{hrs}$ at $37^{\circ} \mathrm{C}$. Then $100 \mu \mathrm{l}$ of culture medium was removed from each well using a manual multichannel pipet, followed by the addition to each well of $100 \mu$ l cell substrate solution (ATPlite kit content). The plate was shaken for $2 \mathrm{~min}$ followed by dark adapted for $10 \mathrm{~min}$ and luminescence was measured using a luminometer (TECAN Genios Pro reader).

E) SYTOX Blue dead cell stain: this is a high-affinity nucleic acid stain that easily penetrates dead cells with compromised plasma membranes but not in the healty ones. Cells were plated in 6 well-plates, incubated in standard medium for $24 \mathrm{hrs}$, and treated with $20 \mathrm{mM}$ metformin for 24 or $48 \mathrm{hrs}$ at $37^{\circ} \mathrm{C}$. After brief incubation 
with SYTOX Blue stain, nucleic acids of dead cells fluoresce bright blue when excited with $405 \mathrm{~nm}$ violet laser light. Samples were analyzed using a Cyan ADP cytofluorimeter (Beckman-Coulter, Brea CA, USA). For each sample, 20,000 events were acquired. The data were analyzed using Summit 4.3.1 software (Beckman-Coulter, USA).

\section{Western blotting}

Cells were plated onto 60-mm dishes for 24 hrs before being treated. Cells were lysed in buffer containing $1 \%$ Nonidet P-40, $20 \mathrm{mM}$ Tris-HCl, pH 8, $137 \mathrm{mM} \mathrm{NaCl}, 10 \%$ glycerol, $2 \mathrm{mM}$ EDTA, $1 \mathrm{mM}$ phenylmethylsulfonyl fluoride, $1 \mathrm{mM}$ sodium orthovanadate, $10 \mathrm{mM} \mathrm{NaF}$ (all from Sigma), and the "Complete" protease inhibitor mixture (Roche Applied Science) for $10 \mathrm{~min}$ at $4^{\circ} \mathrm{C}$ [26]. Nuclei were removed by centrifugation $\left(13,200 \mathrm{rpm}\right.$ at $4^{\circ} \mathrm{C}$, for $2 \mathrm{~min}$ ), and total protein content was measured using the Bradford assay (Bio-Rad). Proteins $(20 \mu \mathrm{g})$ were resuspended in 2X reducing sample buffer (2\% SDS, $62.5 \mathrm{mM}$ Tris, $\mathrm{pH} 6.8,0.01 \%$ bromphenol blue, and $1.43 \mathrm{mM} \beta$ mercaptoethanol, $0.1 \%$ glycerol), electrophoresed on $10 \%$ SDS polyacrylamide gels, transferred on polyvinylidene difluoride membrane (Bio-Rad), and probed with specific antibodies directed against total and phospho-Akt (Ser473), total and phospho-AMPk, total and phosphomTOR, and procaspase- 3 and cleaved caspase- 3 , all purchased from Cell Signaling Technology (Beverly, MA); $\alpha$-tubulin (Sigma) was used as protein loading standard. Immunocomplex detection was performed using ECL system (Bio-Rad), as reported [27].

\section{Real time quantitative RT-PCR analysis}

Total RNAs from samples were extracted using TRIzol reagent (Invitrogen) according to the manufacturer's protocol, DNAseI-digested and subjected to reverse transcription by Transcriptor High Fidelity cDNA Synthesis Kit (Roche 05081955001) following manufacturer's instructions. The total RNA from samples was measured by real-time quantitative RT-PCR using PE ABI PRISM@ 7700 Sequence Detection System (Perkin Elmer Corp./Applied Biosystems, Foster City, CA) and SybrGreen method. The sequences of forward and reverse primers were: NDM29, 5'-GGCAGG CGGGTTCGTT-3' and 5'-CCA CGCCTGGCTAAGTTT TG-3'; neurofilament-68 (NF-68), 5'-CAAGGACGAGGT GTCCG AG-3' and 5'-CCCGGCATGCTTCGA; Integrin beta-1 (CD29), 5'-AACGAGGTCATGGTT CATGTTG-3' and 5'ACCACACCAGCTACAATTGGAA-3'. For endogenous control, the expression of glyceraldehyde 3 phosphate dehydrogenase (GAPDH) gene was examined. The sequences for human GAPDH rRNA forward and reverse primers were 5'-GAAGGTGAAG GTCGGAGTC-3' and 5'-GAAGATGGTGATGGGATTTC-3'. Relative transcript levels were determined from the relative standard curve constructed from stock cDNA dilutions, and divided by the target quantity of the calibrator following manufacturer's instructions.

\section{Statistical analysis}

Experiments were performed at least in triplicate. Data are reported as mean values \pm standard error (SE). Statistical significance of observed differences among different experimental groups was examined using the unpaired Student's t-test, as reported [28]. Statistical analysis were performed before normalization (metformin $v s$ control).

\section{Results}

\section{Metformin inhibits neuroblastoma cell growth}

In order to investigate the effect of metformin on $\mathrm{NB}$, SKNBE2 cells were treated for 12-72 hrs with metformin $(20 \mathrm{mM})$ and proliferation rate analyzed using multiple experimental approaches. First, we measured cell proliferation using the xCELLigence RTCA DP system. SKNBE2 growth curves showed a time-dependent decrease in the proliferation rate of metformin-treated cells, resulting in a statistically significant difference after $48 \mathrm{hrs}$ (as evidenced by the calculated Cell Index, see Methods), and lasting up to the end of the experimental observation (72 hrs) (Figure 1A).

In order to evaluate whether the reduction in Cell Index was directly associated to a decrease of cell proliferation, control and metformin-treated cells were counted using an hemocytometer. Results confirmed a significant reduction of the cell number $48 \mathrm{hrs}$ after metformin administration (Figure 1B). In addition, a statistically significant reduction of the mitotic rate of metformin-treated SKNBE2 cells was confirmed by a statistically significant decrease in DNA synthesis $24 \mathrm{hrs}$ after the treatment, measured by $\left[{ }^{3} \mathrm{H}\right]$-thymidine incorporation (Figure 1C). Next, metformin effects on cell viability were tested using the ATPlite assay, which, measuring intracellular ATP content in metabolically active cells, is an indirect index of cell viability. In agreement with the previously described experiments, we found that, after $48 \mathrm{hrs}$ of treatment, metformin leads to a statistically significant decrease in ATP content (Figure 1D).

To strengthen these results, and verify whether metformin effects were merely cytostatic or cytotoxicity was also induced, we quantified, by FACS analysis, the percentage of dead cells by SYTOX blue incorporation assay, in which the intracellular accumulation of the dye is an index of dead cells. Again, results showed that metformin increased the number of cell death after 48 hrs of treatment (Figure 1E).

In order to assess the mechanism of cell death induced by metformin we tested by Western blot the possible activation of the apoptotic machinery measuring the expression of the cleaved/activated caspase-3. However, 8, 24 and 48 hours of treatment with metformin $20 \mathrm{mM}$ did not increase the cleaved caspase- 3 intracellular content and unaltered caspase-3 pro-enzyme expression was detected 

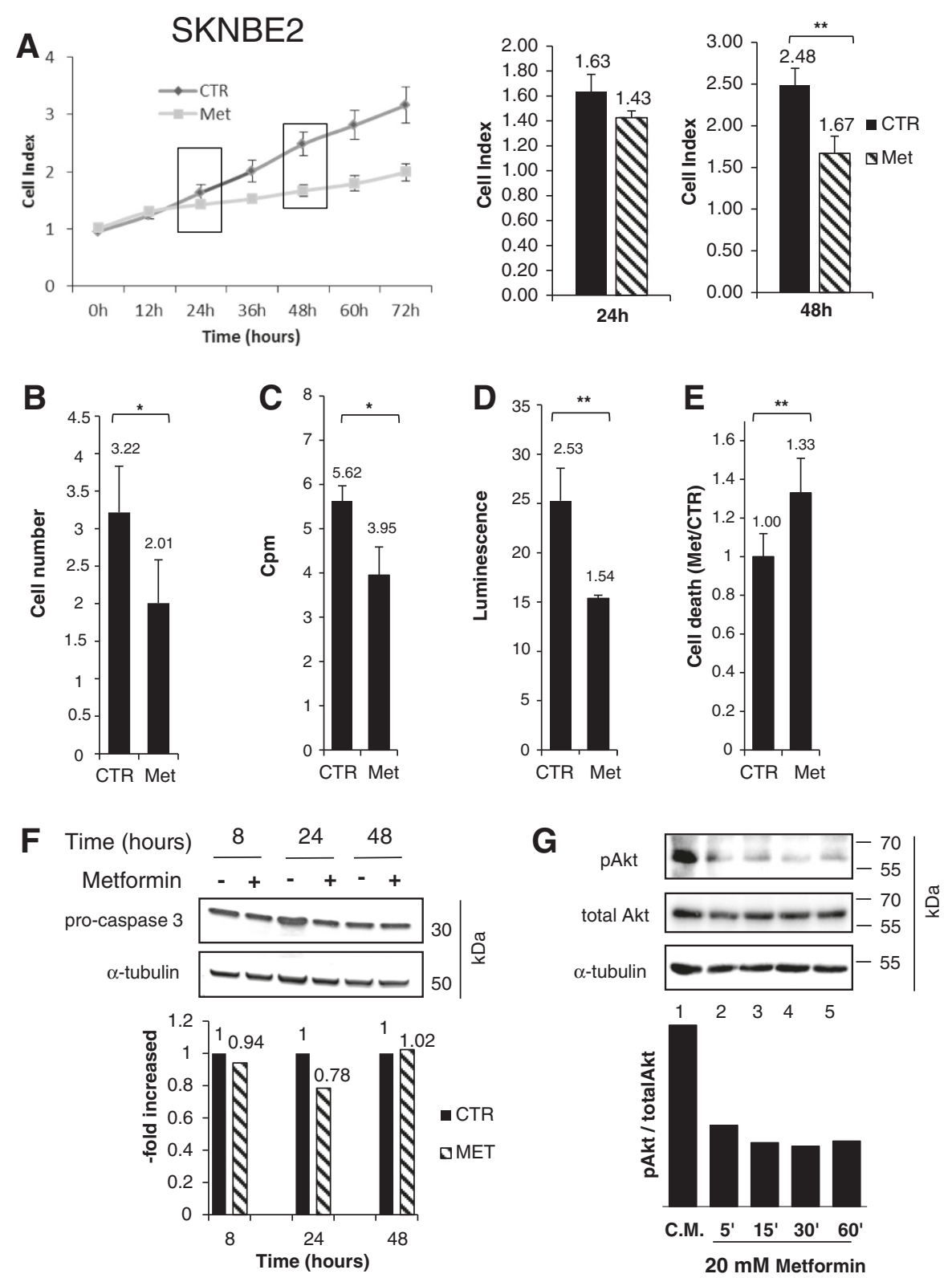

Figure 1 Metformin reduces cell proliferation and viability of Neuroblastoma SKNBE2 cells. A) Cell Index curves reporting SKNBE2 neuroblastoma cell proliferation, untreated (CTR) or treated with metformin (Met), as resulting by Xelligence RTCA DP analysis. Histograms highlight Cell Index values after 24 and 48 hrs. B) Cell counting assay of SKNBE2 cells untreated (CTR) or treated with metformin (Met). Y-axis is referred to cell number $\times 10^{6}$. C) $\left[{ }^{3} \mathrm{H}\right]$-thymidine incorporation assay of SKNBE2 cells, untreated (CTR) or treated with metformin (Met). Y-axis is referred to $\mathrm{cpm} \times 10^{3}$. D) Cell viability analysis of SKNBE2 cells untreated (CTR) or treated with metformin (Met) as determined by ATPlite assay. Y-axis is referred to luminescence $\times 10^{5}$. E) Cell death analysis by FACS analysis for sytox blue staining. The percentage of cell death is reported as Met/CTR ratio. F) Western blot analysis of caspase-3 cleavage. Equal loading of proteins was ensured by normalization for pro-caspase 3 and atubulin expression. Cleaved Caspase 3 was not detected. G) Western blot analysis of Akt phosphorylation/activation. $1=$ Complete Medium (C.M., RPMI 10\%), $2=$ =.M. +20 mM Met for 5 min, $3=$ =.M. +20 mM Met for 15 min, $4=$ C.M. +20 mM Met for 30 min, $5=$ C.M. + 20 mM Met for $60 \mathrm{~min}$. Equal loading of proteins was ensured by normalization for total Akt and a-tubulin expression. Densitometric analysis of phospho-Akt/ total Akt ratio is also reported. ( $\left.{ }^{*} p<0.05 ;{ }^{* *} p<0.01\right)$.

(Figure 1F). In addition, in treated cells, we observed neither the up-regulation of the pro-apoptotic protein Bax, nor the presence of nuclear piknosis, both common features of apoptotic cells (data not shown). Altogether these results exclude apoptosis from the major causes of cell death induced by metformin.

To investigate at molecular level the intracellular pathways altered by metformin to affect SKNBE2 cells 
proliferation and survival, we measured its effects on Akt phosphorylation/activation. Akt inhibition, known to correlate with impaired cell survival, was recently shown to represent a molecular correlate of metformin direct antitumoral effects [15]. In Western blot experiments, we report that metformin $(20 \mathrm{mM})$ induced a long-lasting (5-60 min) decline in Akt phosphorylation/activation (Figure 1G), suggesting that metformin effects in SKNBE2 cells were mediated by the inhibition of Akt activity. Conversely, AMPK/mTOR pathways was not affected (see below).

Since malignant potential of NB nodules is often dependent on N-myc amplification/overexpression (as occurs in SKNBE2 cells), we investigated whether the antitumoral effects of metformin are somehow modulated by the activity of this oncogene. To this purpose we tested metformin antiproliferative activity in the SH-SY5Y NB cell line in which N-myc is not amplified [29]. Metformin $(20 \mathrm{mM})$ treatment induced a statistically significant reduction of proliferation rate after $48 \mathrm{hrs}$, also in SH-SY5Y cells (Figure 2A). In addition, metformin effect on cell viability was assessed by ATPlite assay, performed after 24 and 48 hrs of treatment. A reduction of cell viability was detected although to a lesser extent respect to SKNBE2 cells, reaching statistical significance after 48 hrs of treatment (Figure 2B). Interestingly, after $48 \mathrm{hrs}$ of treatment, SYTOX blue assay evidenced that metformin-induced cell death in SH-SY5Y cells, although reaching statistical significance, was less pronounced as compared to what observed in SKNBE2 cells (Figure 2C).
Altogether these results suggest that metformin is an effective antiproliferative agent towards NB cell lines with different malignant potential.

\section{Effects of metformin on differentiated neuroblastoma cell growth}

$\mathrm{NB}$ is composed by extremely heterogeneous cell populations, including cells at different maturation stages. Thus, we investigated the efficacy of metformin as antiproliferative agent in NB cells at different level of differentiation and malignancy.

To this aim we first assessed the effects of the treatment with metformin using SKNBE2 cells differentiated after ATRA treatment $(10 \mu \mathrm{M}$, for 10 days $)$ that leads to a neuron-like commitment (Figure 3A). Interestingly, in cell count experiments, we found that the remarkable reduction of cell proliferation rate induced by metformin in non-differentiated control cells, was greatly reduced in differentiated SKNBE cells exposed to metformin, resulting in a highly significant statistical difference comparing the two experimental groups (Figure 3B). SYTOX blue assay corroborated this evidence showing, after metformin treatment, a significant increase of death rate in non-differentiated cells, which was almost abolished in SKNBE2 cells differentiated with ATRA (Figure 3C). In order to discriminate between the antiproliferative effect that ATRA exert per se and those induced by metformin we treated SKNBE2 cells with a lower dose of ATRA ( $1 \mu \mathrm{M}$, for 10 days). In this condition the treatment induced slight morphological changes accompanied by a

\section{SH-SY5Y}
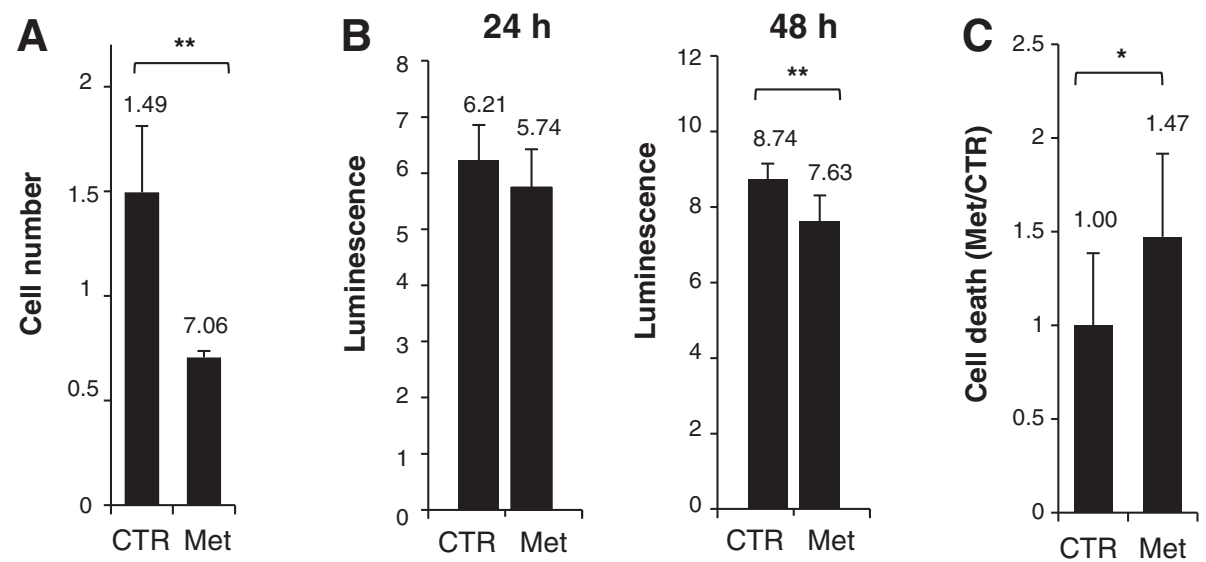

Figure 2 Metformin antiproliferative effects towards neuroblastoma cell lines with different malignant potential. A) Cell counting assay in SH-SY5Y cells, untreated (CTR) or treated with metformin (Met). Y-axis is referred to cell number $\times 10^{6}$. B) Cell viability analysis of SH-SY5Y cells untreated (CTR) or treated with metformin (Met), as determined by ATPlite assay. Y-axis is referred to luminescence $\times 10^{5}$. C) Cell death quantification by FACS analysis for SYTOX blue staining in untreated (CTR) or metformin (Met)-treated cells. The percentage of cell death is reported as Met/CTR ratio. $\left({ }^{*} p<0.05 ; * * 0.01\right)$. 


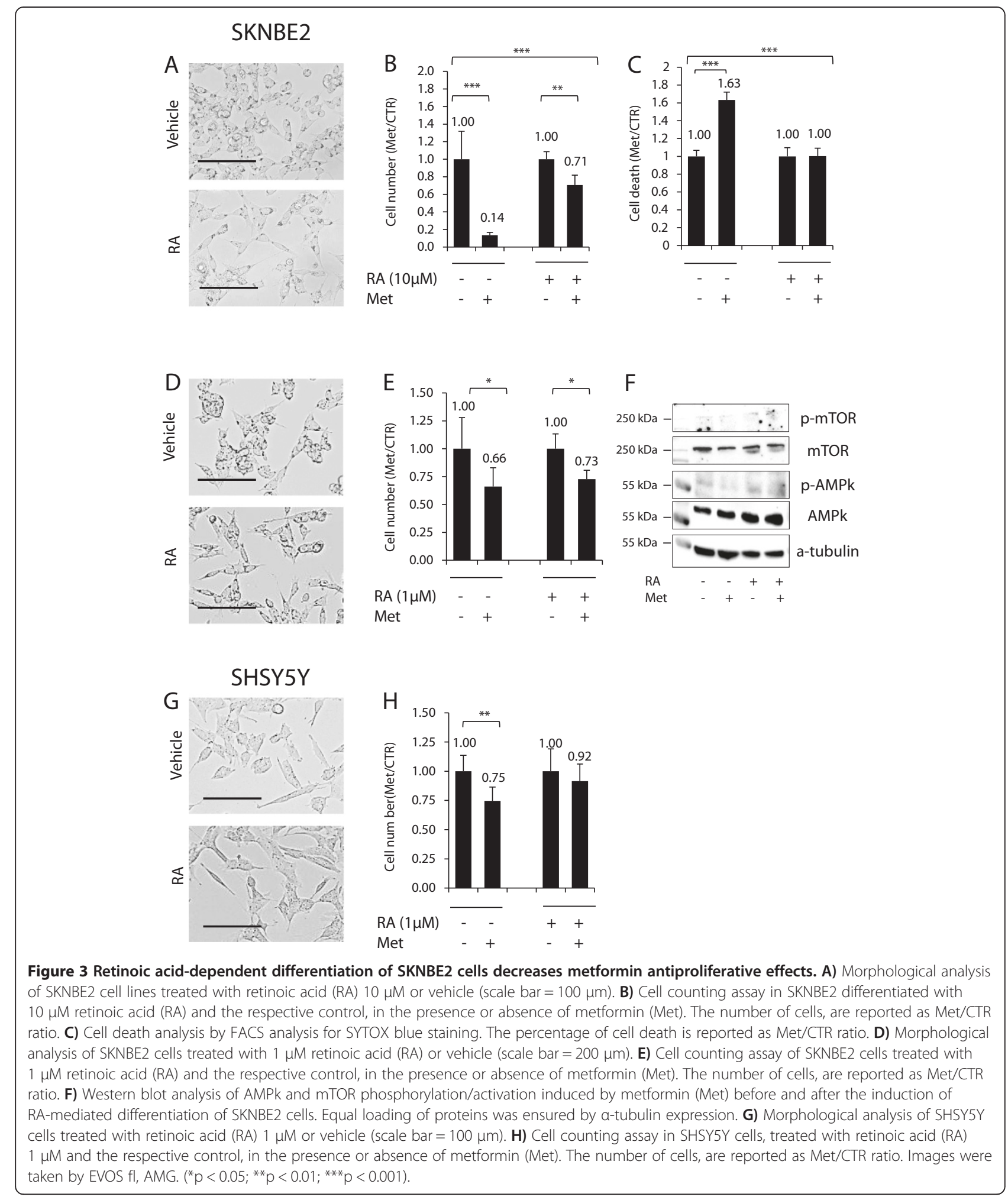

weak reduction of metformin susceptibility as showed by cell counting experiments (Figure 3D,E). Therefore, ATRA affects metformin susceptibility in SKNBE cells by inducing NB cells differentiation in a dose-dependent manner.
Since in differentiated hepatic cells metformin hypoglycemic effects are mainly mediated by the modulation of the AMPK/mTOR pathway, we examined whether this may occur also in differentiated NB cells. By western blot 
analysis we compared the effects of metformin on AMPK and mTOR phosphorylation before and after the induction of ATRA-mediated differentiation in SKNBE2 cells. As reported in Figure 3F, no differences on the activity (phosphorylation) of AMPk or mTOR has been found in both differentiated and undifferentiated cells. Altogether, these results show that the susceptibility to the effects of metformin is directly correlated to NB differentiation level, as reported in glioblastoma cells [15] and confirmed that AMPK seems not involved in the antiproliferative effects of the drug.

Next we investigated the occurrence of this effect in NB cells highly responsive to ATRA-induced differentiation. We found that, although less sensitive to metformin than SKNBE2 cells, SHSY5Y cells were highly influenced by ATRA treatment ( $1 \mu \mathrm{M}$, for 10 days) that completely abolished the ability of metformin to reduce cell proliferation (Figure 3G,H). Higher ATRA concentrations $(10 \mu \mathrm{M}$, for 10 days) did not lead to additional changes in metformin susceptibility (data not shown).
To delve deeper into the mechanisms by which differentiation may affect metformin antiproliferative effects in NB cells, we took advantage of a novel in vitro model of NB differentiation, we recently developed, that was based on the expression level of a nc-RNA, named NDM29, in SKNBE2 cells $[21,22,30]$. In this system the level of differentiation of NB cells, as well as the reduction of the stemness/tumor initiating potential, is directly related to the level of synthesis of NDM29 [21,22,30]. Here, we compared the effects of metformin in mock transfected cells, that express low levels of endogenous NDM29 RNA, with established cell lines in which different levels of NDM29 are overexpressed. The overexpression of ncRNA NDM29 represent a novel model of NB cell differentiation, resulting in the acquisition of neuronal electrophysiological properties as previously reported [22].

In particular, we used the following permanently transfected cell lines: SKNBE2 cells that express NDM29 at endogenous level (transfected with the empty vector, pMock), NDM29 overexpressing cells, showing increased
A SKNBE2-Mock
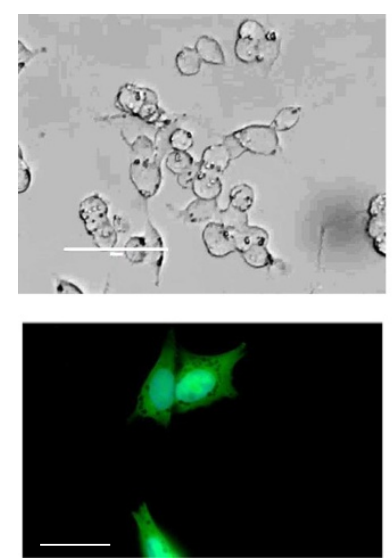

D

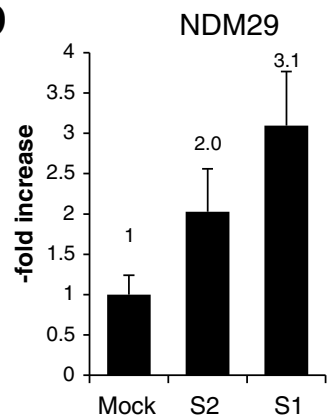

B SKNBE2-S2
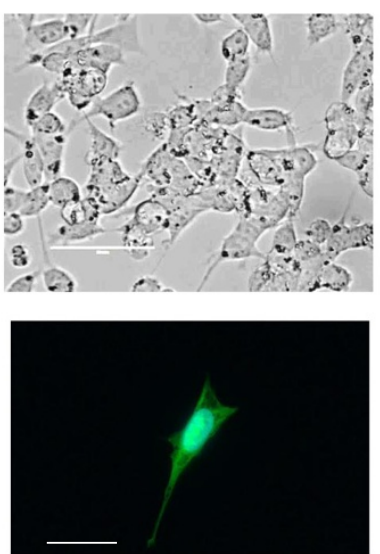

NF68

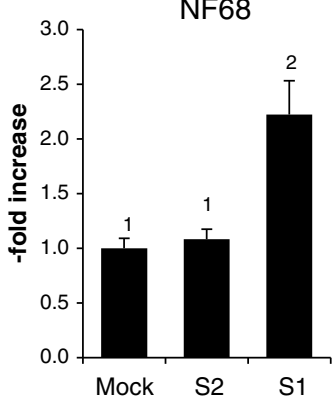

\section{SKNBE2-S1}
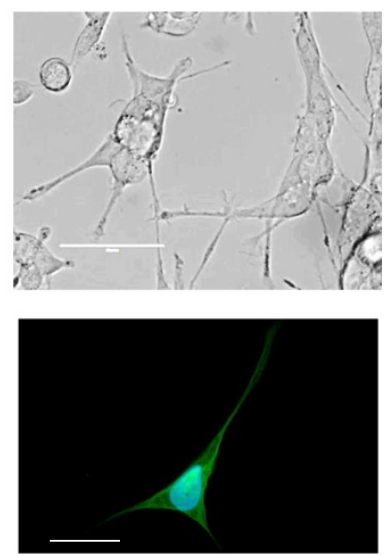

CD29

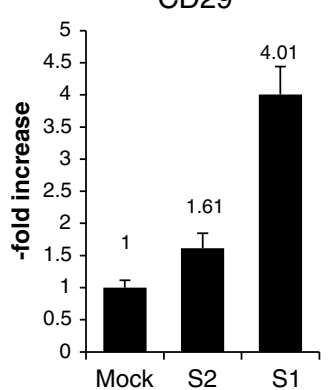

Figure 4 NDM29 expression level correlates with the differentiation of NB cells toward a neuron-like phenotype. A) SKNBE2-Mock cells expressing the basal level of NDM29 ncRNA; B,C) SKNBE2-S2 and SKNBE2-S1 cells expressing increased level of NDM29 [21]; (scale bar = 50 $\mu$ m). Upper panels: transmitted light microscopy (EVOS fl, AMG). Lower panels: fluorescence microscopy (Zeiss Axiovert 200 M inverted microscope, Jena Germany). Blue = DAPI, green = GFP (scale bar $=25 \mu \mathrm{m}$ ). D) Real time RT-PCR analysis of NDM29, NF-68, (differentiation markers) and CD29 (adhesion marker) expression. 


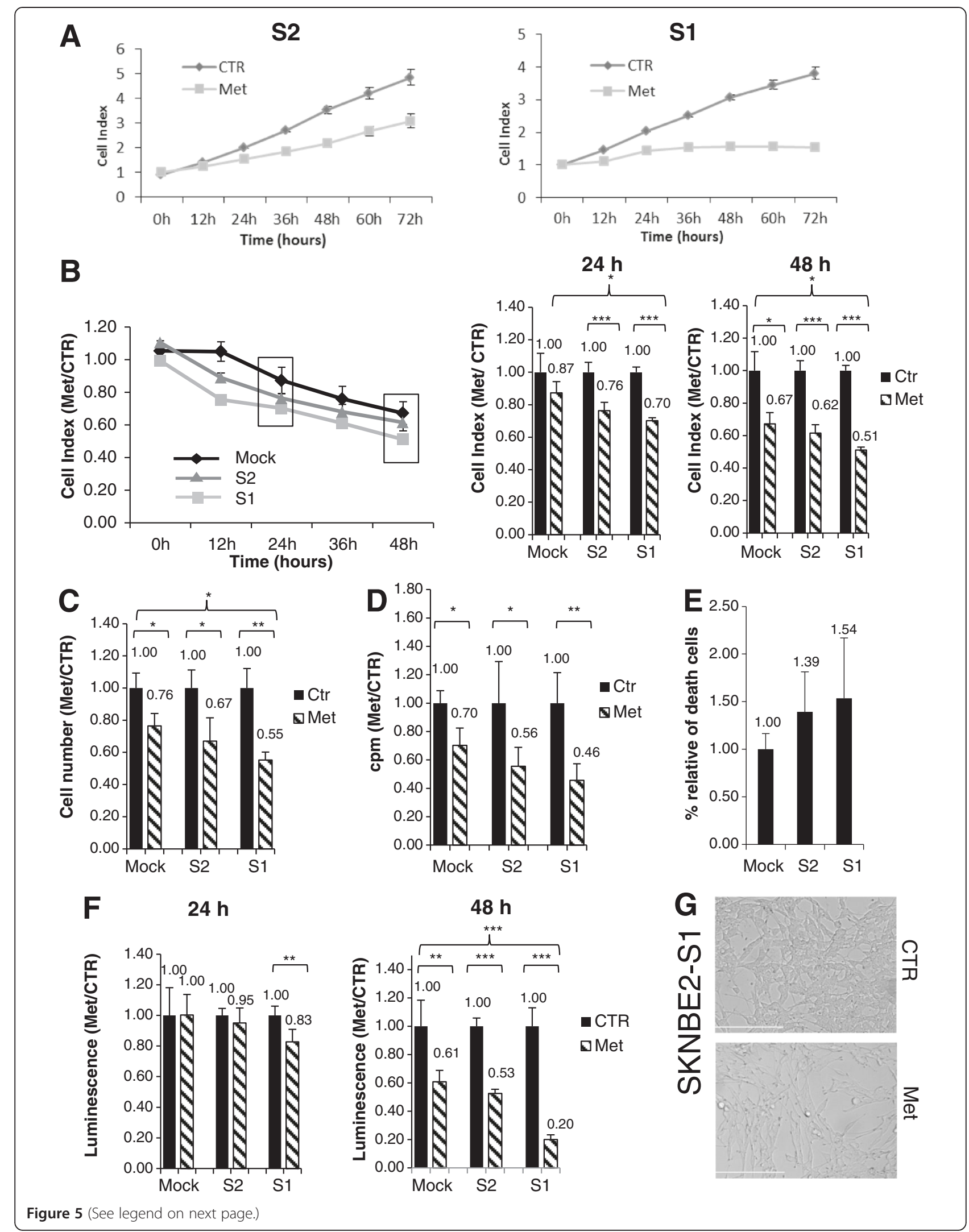



analysis. B) Cell Index analysis of SKNBE2-Mock, SKNBE2-S2 and SKNBE2-S1 NB cells untreated (CTR) or treated with metformin (Met), as resulting by Xcelligence RTCA DP analysis. Data were reported as Met/CTR ratio for each cell line. Histogram on the right panel report statistical analysis of 24 and 48 hrs cell index measures. C) Cell counting assay and D) [ ${ }^{3} \mathrm{H}$ ]-thymidine incorporation assay of SKNBE2-Mock, SKNBE2-S2 and SKNBE2-S1 cells untreated (CTR) or treated with metformin (Met). Cell number (C) and cpm (D) values are reported as Met/CTR ratio for each cell type. E) FACS analysis of cell dead with SYTOX blue stain of different cell lines (SKNBE2-Mock, S2, S1). The percentage of cell death is reported as Met/CTR ratio, for each cell type. F) Cell viability determination by ATPlite assay in different cell lines (SKNBE2-Mock, S2 and S1) untreated (CTR) or treated with metformin (Met). Luminescence values, are reported as Met/CTR ratio for each cell type. G) Morphology of S1 cells (scale bar $=200 \mu \mathrm{m})$ by EVOS fl, AMG. $\left({ }^{*} \mathrm{p}<0.05 ;{ }^{* *} \mathrm{p}<0.01 ;{ }^{* *} \mathrm{p}<0.001\right)$.

NDM29 RNA expression 2- (S2) and 3.1-fold (S1) over pMock cells. As depicted in Figure 4, NDM29 stably transfected cells, co-expressing GFP, show a strong enrichment in neuritic processes, acquiring a frank neuronal morphology, associated to the expression of neuronal (neurofilament NF-68) and adhesion (CD29) markers. Importantly, the acquisition of all the differentiation features were proportional to the expression level of NDM29 (Figure 4A-D). Cells from all these lines were treated for $48 \mathrm{hrs}$ with metformin $(20 \mathrm{mM})$ and tested for cell proliferation rate. Surprisingly, by Xcelligence detection system, we observed that metformin was able to affect cell proliferation also in the differentiated $\mathrm{S} 2$ and $\mathrm{S} 1$ cell lines (Figure 5A). Interestingly, we found that metformin activity was directly correlated to NDM29 expression, which, in turn, determines the differentiation level of the cells. Indeed, higher metformin-dependent inhibition of cell proliferation was observed in well-differentiated S1 cells than in mock-transfected cells (Figure 5B). In order to better associate these effects to cell proliferation rather than an artifactual parameter detected by this system, such as cell area, these data were confirmed in cell counting and $\left[{ }^{3} \mathrm{H}\right]$ thymidine incorporation experiments showing that metformin inhibition of DNA synthesis showed a higher statistical significance in NDM29-overexpressing cells as compared to mock cells (Figure 5C,D). In agreement with these results, FACS analysis for SYTOX blue staining, after 24 hours of metformin treatment, showed increased SKNBE2 death rate directly correlated with NDM29 expression (Figure 5E), and ATPlite assay showed decreased cell viability, proportional to the levels of NDM29 expression (Figure 5F). Interestingly, microscopic examination revealed a dramatic alteration in the morphology of metformin-treated S1 cells that acquired large, elongated shape recapitulating a behavior previously observed in glioma cell lines (Figure 5G) [31,32].

These results highlight an unexpected different modulation of the response to metformin after NB cell differentiation, which is not correlated to differentiation per se but directly depend on the expression level of NDM29 ncRNA.

We previously reported that NDM29 sensitizes SKNBE2 cells to several cytotoxic drugs (cisplatin, doxorubicin) by powerfully down-regulating MDR1 expression [21]. Thus, we verified whether the increased sensitivity of metformin observed in S1 cells might be related to NDM29dependent alterations of the expression of molecules involved in the metformin interaction with the cells. In particular, we focused on the expression of the organic cation transporter 1 (OCT-1) that represents one of the main regulators of metformin cell internalization. However, in Western blot analysis we did not find significant differences in OCT-1 expression in pMock, S1 and S2 cells (data not shown), suggesting that different mechanisms should be responsible of the higher sensitivity to metformin of NDM29 expressing cells. Conversely, we observed that in SH-SY5Y OCT-1 expression was slightly reduced after metformin treatment (data not shown), possibly representing the molecular correlate for the lower sensitivity of these cells as compared to SKBNE2.

\section{Discussion}

Besides being a first-line antidiabetic drug, metformin is currently under consideration for additional anticancer properties [33-35]. Recent reports evidenced TICs from different cancer types as the preferential targets of this drug [14-16]. These studies are in line with reports showing that also other antidiabetic drugs, such as PPAR- $\gamma$ agonists exert cytostatic effects [36,37].

In this study we report, for the first time, an antiproliferative effect of metformin in a high-risk NB cell model. We show that metformin induces a significant reduction in the proliferation rate in two different $\mathrm{NB}$ cell lines (SKNBE2 and SH-SY5Y) characterized by different N-myc expression, although a higher sensitivity was observed in the first one. While we cannot provide a definitive answer for this difference, the observation that in SH-SY5Y cells, OCT-1 expression, which control the cellular internalization of this metformin, is down-regulated upon metformin treatment, could account for this difference.

In line with previous studies [15], this effect was related to a powerful inhibition of Akt activation, suggesting that the treatment with metformin could directly act on cell viability. In fact, beside an effect on cell cycle progression, as suggested by the reduction of DNA synthesis in $\left[{ }^{3} \mathrm{H}\right]$ thymidine incorporation experiments, also increased cell death was observed in SYTOX blue staining experiments. 
Interestingly, metformin effects on cell survival did not were the results of activation of apoptosis since we did not detect nuclear shrinkage, caspase 3 activation or Bax upregulation. Thus, from our data metformin elicits mainly a cytostatic effect that could indirectly cause cell death via apoptosis-independent pathways.

Interestingly, no changes in AMPK activity was observed in NB cells, although this kinase was reported to represent a key intracellular mechanism in metformin effects [38]. However, several studies suggested that mechanisms, others than AMPK activation, could mediate antiproliferative activity of metformin [39-41]. In particular, although several different mechanisms were identified in different tumoral cells, down-regulation of IGF-1 or other growth factor mediated autocrine signalling was suggested as main antiproliferative mechanisms regulated by metformin $[42,43]$. In turn, this effect could be responsible for the inhibition of pro-survival intracellular pathways, such as Akt, as we show in this study in NB cells and previously reported in GBM TICs [15]. However, further studies are still required to definitely address this issue.

Importantly, as shown in several other tumor cell types $[14,15]$, metformin antitumoral activity was significantly higher in less differentiated NB cells, with a lower effect in differentiated cells (here we used ATRAdependent differentiation).

Conversely, we found that metformin sensitivity is highly increased in NB cells in which differentiation is induced by NDM29 overexpression. This effect was strictly dependent on the expression levels of this ncRNA, which also correlate with the induction of NB cell differentiation. These data, and in particular the direct relationship between NDM29 expression and the reduction of NB cell viability induced by metformin, strongly suggest the possibility of a causal effect between the two events. At present we do not know the exact molecular mechanisms by which NDM29-dependent differentiation increases NB cell susceptibility to metformin, at odd with ATRA effects. In particular, since we previously reported that NDM29 overexpression inhibits MDR1 expression, representing a molecular mechanism that increases NB cell vulnerability to antimitotic cytotoxic drugs [21], we hypothesized that NDM29 expression might affect susceptibility to metformin inducing OCT-1 expression, thus increasing the amount of drug internalized into the cells and able to interfere with survival signals, such as Akt activity. However, here we report that SKBNE2 cells express similar OCT-1 content independently from the levels of NDM29 expression, and thus a different molecular mechanism needs to be identified.

\section{Conclusions}

Our study for the first time demonstrates that metformin exerts antitumor activity against high risk NB cells, reducing cell proliferation and viability, via inhibition of Akt phosphorylation, showing higher sensitivity for less differentiated, highly proliferative cells. These data represent the starting point for further studies aimed to test the possible application of metformin in NB therapy. Moreover, as already shown for other cytotoxic drugs, the overexpression of NDM29, although inducing neuronal differentiation, powerfully sensitizes NB cells to metformin antiproliferative effects, suggesting that the pharmacological modulation of the expression of this ncRNA may represent a potential goal in the NB therapy. The molecular determinants by which the differentiation induced by NDM29, but not by retinoic acid, increases the antiproliferative activity of metformin will also represent a future goal in the translational research of novel NB therapies.

\section{Competing interests}

The authors declare that they have no competing interests.

\section{Authors' contributions}

DC and AG made the vast majority of the experiments here reported and participated to the interpretation of results. RW performed Western blot analysis. TF contributed to the planning the experiments and the interpretation and discussion of the results and the draft of the manuscript. RC participated to the discussion of the results. AP planned the experiments, interpreted the results and wrote the manuscript. All authors read and approved the final manuscript.

\section{Authors' information}

Delfina Costa and Arianna Gigoni share first authorship.

\section{Acknowledgments}

A.P. was supported by the Associazione Italiana Ricerca sul Cancro (2009 AIRC Program n IG9378), by the IRCCS-AOU San Martino-IST, Genova-Italy (Progetto $5 \times 1000$ ) and by the Associazione Italiana per la Lotta al Neuroblastoma/Fondazione Neuroblastoma (Genoa, Italy).

\section{Author details}

${ }^{1}$ Department of Experimental Medicine (DIMES), University of Genova, Genova, Italy. ${ }^{2}$ Internal Medicine (DIMI), University of Genova, Genova, Italy. ${ }^{3}$ IRCCS-AOU San Martino-IST, Genova, Italy. ${ }^{4}$ Center of Excellence for Biomedical Research (CEBR), University of Genova, Genova, Italy.

Received: 25 February 2014 Accepted: 4 June 2014

Published: 2 July 2014

\section{References}

1. Ben Sahra I, Le Marchand-Brustel Y, Tanti JF, Bost F: Metformin in cancer therapy: a new perspective for an old antidiabetic drug? Mol Cancer Ther 2010, 9:1092-1099.

2. Zhou G, Myers R, Li Y, Chen Y, Shen X, Fenyk-Melody J, Wu M, Ventre J, Doebber T, Fujii N, Musi N, Hirshman MF, Goodyear L, Moller DE: Role of AMP-activated protein kinase in mechanism of metformin action. J Clin Invest 2001, 108:1167-1174.

3. Decensi A, Puntoni M, Goodwin P, Cazzaniga M, Gennari A, Bonanni B, Gandini S: Metformin and cancer risk in diabetic patients: a systematic review and meta-analysis. Cancer Prev Res (Phila) 2010, 3:1451-1461.

4. Zhang P, Brown MB, Bilik D, Ackermann RT, Li R, Herman WH: Health utility scores for people with type 2 diabetes in U.S. managed care health plans: results from Translating Research Into Action for Diabetes (TRIAD). Diabetes Care 2012, 35:2250-2256.

5. Col NF, Ochs L, Springmann V, Aragaki AK, Chlebowski RT: Metformin and breast cancer risk: a meta-analysis and critical literature review. Breast Cancer Res Treat 2012, 135:639-646. 
6. Margel D, Urbach DR, Lipscombe LL, Bell CM, Kulkarni G, Austin PC, Fleshner $\mathrm{N}$ : Metformin Use and All-Cause and Prostate Cancer-Specific Mortality Among Men With Diabetes. J Clin Oncol 2013, 31:3069-3075.

7. Jiralerspong S, Palla SL, Giordano SH, Meric-Bernstam F, Liedtke C, Barnett CM, Hsu L, Hung MC, Hortobagyi GN, Gonzalez-Angulo AM: Metformin and pathologic complete responses to neoadjuvant chemotherapy in diabetic patients with breast cancer. J Clin Oncol 2009, 27:3297-3302.

8. Algire C, Zakikhani M, Blouin MJ, Shuai JH, Pollak M: Metformin attenuates the stimulatory effect of a high-energy diet on in vivo LLC1 carcinoma growth. Endocr Relat Cancer 2008, 15:833-839.

9. Ben Sahra I, Laurent K, Loubat A, Giorgetti-Peraldi S, Colosetti P, Auberger P, Tanti JF, Le Marchand-Brustel Y, Bost F: The antidiabetic drug metformin exerts an antitumoral effect in vitro and in vivo through a decrease of cyclin D1 level. Oncogene 2008, 27:3576-3586.

10. Kisfalvi K, Eibl G, Sinnett-Smith J, Rozengurt E: Metformin disrupts crosstalk between $\mathrm{G}$ protein-coupled receptor and insulin receptor signaling systems and inhibits pancreatic cancer growth. Cancer Res 2009, 69:6539-6545.

11. Zakikhani M, Dowling RJ, Sonenberg N, Pollak MN: The effects of adiponectin and metformin on prostate and colon neoplasia involve activation of AMPactivated protein kinase. Cancer Prev Res (Phila) 2008, 1:369-375.

12. Hosono K, Endo H, Takahashi H, Sugiyama M, Sakai E, Uchiyama T, Suzuki K, lida H, Sakamoto Y, Yoneda K, Koide T, Tokoro C, Abe Y, Inamori M, Nakagama H, Nakajima A: Metformin suppresses colorectal aberrant crypt foci in a short-term clinical trial. Cancer Prev Res (Phila) 2010, 3:1077-1083.

13. Hadad S, Iwamoto T, Jordan L, Purdie C, Bray S, Baker L, Jellema G, Deharo S, Hardie DG, Pusztai L, Moulder-Thompson S, Dewar JA, Thompson AM: Evidence for biological effects of metformin in operable breast cancer: a pre-operative, window-of-opportunity, randomized trial. Breast Cancer Res Treat 2011, 128:783-794.

14. Hirsch HA, lliopoulos D, Tsichlis PN, Struhl K: Metformin selectively targets cancer stem cells, and acts together with chemotherapy to block tumor growth and prolong remission. Cancer Res 2009, 69:7507-7511.

15. Würth R, Pattarozzi A, Gatti M, Bajetto A, Corsaro A, Parodi A, Sirito R, Massollo M, Marini C, Zona G, Fenoglio D, Sambuceti G, Filaci G, Daga A Barbieri F, Florio T: Metformin selectively affects human glioblastoma tumor-initiating cell viability: A role for metformin-induced inhibition of Akt. Cell Cycle 2013, 12:145-156.

16. Saito T, Chiba T, Yuki K, Zen Y, Oshima M, Koide S, Motoyama T, Ogasawara S, Suzuki E, Ooka Y, Tawada A, Tada M, Kanai F, Takiguchi Y, Iwama A, Yokosuka O: Metformin, a diabetes drug, eliminates tumor-initiating hepatocellular carcinoma cells. PLoS One 2013, 8:e70010.

17. Würth R, Barbieri F, Florio T: New molecules and old drugs as emerging approaches to selectively target human glioblastoma cancer stem cells. BioMed Res Int 2014, 2014:126586.

18. Florio T, Barbieri F: The status of the art of human malignant glioma management: the promising role of targeting tumor-initiating cells. Drug Discov Today 2012, 17:1103-1110.

19. Del Barco S, Vazquez-Martin A, Cufi S, Oliveras-Ferraros C, Bosch-Barrera J, Joven J, Martin-Castillo B, Menendez JA: Metformin: multi-faceted protection against cancer. Oncotarget 2011, 2:896-917.

20. Rattan R, Ali Fehmi R, Munkarah A: Metformin: an emerging new therapeutic option for targeting cancer stem cells and metastasis. J Oncol 2012, 2012:928127.

21. Castelnuovo M, Massone S, Tasso R, Fiorino G, Gatti M, Robello M, Gatta E, Berger A, Strub K, Florio T, Dieci G, Cancedda R, Pagano A: An Alu-like RNA promotes cell differentiation and reduces malignancy of human neuroblastoma cells. Faseb J 2010, 24:4033-4046.

22. Gavazzo P, Vella S, Marchetti C, Nizzari M, Cancedda R, Pagano A: Acquisition of neuron-like electrophysiological properties in neuroblastoma cells by controlled expression of NDM29 ncRNA. J Neurochem 2011, 119:989-1001.

23. Massone S, Ciarlo E, Vella S, Nizzari M, Florio T, Russo C, Cancedda R, Pagano A: NDM29, a RNA polymerase III-dependent non coding RNA, promotes amyloidogenic processing of APP and amyloid beta secretion. Biochim Biophys Acta 2012, 1823:1170-1177.

24. Penna I, Vassallo I, Nizzari M, Russo D, Costa D, Menichini P, Poggi A, Russo C, Dieci G, Florio T, Cancedda R, Pagano A: A novel snRNA-like transcript affects amyloidogenesis and cell cycle progression through perturbation of Fe65L1 (APBB2) alternative splicing. Biochim Biophys Acta 2013, 1833:1511-1526.

25. Galante D, Corsaro A, Florio T, Vella S, Pagano A, Sbrana F, Vassalli M, Perico A, D'Arrigo C: Differential toxicity, conformation and morphology of typical initial aggregation states of Abeta1-42 and Abetapy3-42 beta-amyloids. Int J Biochem Cell Biol 2012, 44:2085-2093.
26. Pattarozzi A, Gatti M, Barbieri F, Wurth R, Porcile C, Lunardi G, Ratto A, Favoni R, Bajetto A, Ferrari A, Florio T: 17beta-estradiol promotes breast cancer cell proliferation-inducing stromal cell-derived factor-1-mediated epidermal growth factor receptor transactivation: reversal by gefitinib pretreatment. Mol Pharmacol 2008, 73:191-202.

27. Pagano A, Castelnuovo M, Tortelli F, Ferrari R, Dieci G, Cancedda R: New small nuclear RNA gene-like transcriptional units as sources of regulatory transcripts. PLoS Genet 2007, 3:e1.

28. Ciarlo E, Massone S, Penna I, Nizzari M, Gigoni A, Dieci G, Russo C, Florio T, Cancedda R, Pagano A: An intronic ncRNA-dependent regulation of SORL1 expression affecting Abeta formation is upregulated in post-mortem Alzheimer's disease brain samples. Dis Model Mech 2013, 6:424-433.

29. Walton JD, Kattan DR, Thomas SK, Spengler BA, Guo HF, Biedler JL, Cheung NK, Ross RA: Characteristics of stem cells from human neuroblastoma cell lines and in tumors. Neoplasia 2004, 6:838-845.

30. Gavazzo P, Vassalli M, Costa D, Pagano A: Novel ncRNAs transcribed by Pol III and elucidation of their functional relevance by biophysical approaches. Front Cell Neurosci 2013, 7:203.

31. Janjetovic K, Harhaji-Trajkovic L, Misirkic-Marjanovic M, Vucicevic L, Stevanovic D, Zogovic N, Sumarac-Dumanovic M, Micic D, Trajkovic V: In vitro and in vivo anti-melanoma action of metformin. Eur J Pharmacol 2011, 668:373-382.

32. Isakovic A, Harhaji L, Stevanovic D, Markovic Z, Sumarac-Dumanovic M, Starcevic $V$, Micic D, Trajkovic V: Dual antiglioma action of metformin: cell cycle arrest and mitochondria-dependent apoptosis. Cell Mol Life Sci 2007, 64:1290-1302

33. Correia S, Carvalho C, Santos MS, Proenca T, Nunes E, Duarte Al, Monteiro P, Seica R, Oliveira CR, Moreira PI: Metformin protects the brain against the oxidative imbalance promoted by type 2 diabetes. Med Chem 2008, 4:358-364.

34. Correia S, Carvalho C, Santos MS, Seica R, Oliveira CR, Moreira PI: Mechanisms of action of metformin in type 2 diabetes and associated complications: an overview. Mini Rev Med Chem 2008, 8:1343-1354

35. Gonzalez-Angulo AM, Meric-Bernstam F: Metformin: a therapeutic opportunity in breast cancer. Clin Cancer Res 2010, 16:1695-1700.

36. Vitale G, Zappavigna S, Marra M, Dicitore A, Meschini S, Condello M, Arancia G, Castiglioni S, Maroni P, Bendinelli P, Piccoletti R, van Koetsveld PM, Cavagnini F, Budillon A, Abbruzzese A, Hofland LJ, Caraglia M: The PPARgamma agonist troglitazone antagonizes survival pathways induced by STAT-3 in recombinant interferon-beta treated pancreatic cancer cells. Biotechnol Adv 2012, 30:169-184.

37. Dicitore A, Caraglia M, Gaudenzi G, Manfredi G, Amato B, Mari D, Persani L, Arra C, Vitale G: Type I interferon-mediated pathway interacts with peroxisome proliferator activated receptor-gamma (PPAR-gamma): at the cross-road of pancreatic cancer cell proliferation. Biochim Biophys Acta 2014, 1845:42-52.

38. Russo GL, Russo M, Ungaro P: AMP-activated protein kinase: a target for old drugs against diabetes and cancer. Biochem Pharmacol 2013, 86:339-350.

39. Memmott RM, Mercado JR, Maier CR, Kawabata S, Fox SD, Dennis PA: Metformin prevents tobacco carcinogen-induced lung tumorigenesis. Cancer Prev Res (Phila) 2010, 3:1066-1076.

40. Sarfstein R, Friedman Y, Attias-Geva Z, Fishman A, Bruchim I, Werner H: Metformin downregulates the insulin/IGF-I signaling pathway and inhibits different uterine serous carcinoma (USC) cells proliferation and migration in p53-dependent or -independent manners. PLoS One 2013, 8:e61537.

41. Liu X, Chhipa RR, Pooya S, Wortman M, Yachyshin S, Chow LM, Kumar A, Zhou X, Sun Y, Quinn B, McPherson C, Warnick RE, Kendler A, Giri S, Poels J, Norga K, Viollet B, Grabowski GA, Dasgupta B: Discrete mechanisms of mTOR and cell cycle regulation by AMPK agonists independent of AMPK. Proc Natl Acad Sci U S A 2014, 111:E435-E444.

42. Quinn BJ, Dallos M, Kitagawa H, Kunnumakkara AB, Memmott RM, Hollander MC, Gills JJ, Dennis PA: Inhibition of lung tumorigenesis by metformin is associated with decreased plasma IGF-I and diminished receptor tyrosine kinase signaling. Cancer Prev Res (Phila) 2013, 6:801-810.

43. Li L, Han R, Xiao H, Lin C, Wang Y, Liu H, Li K, Chen H, Sun F, Yang Z, Jiang J, He Y: Metformin Sensitizes EGFR-TKI-Resistant Human Lung Cancer Cells In Vitro and In Vivo through Inhibition of IL-6 Signaling and EMT Reversal. Clin Cancer Res 2014, 20:2714-2726.

doi:10.1186/1475-2867-14-59

Cite this article as: Costa et al:: Metformin inhibition of neuroblastoma cell proliferation is differently modulated by cell differentiation induced by retinoic acid or overexpression of NDM29 non-coding RNA. Cancer Cell International 2014 14:59. 